\title{
Combining experimental and numerical analysis to estimate stress fields along the surface crack front
}

\author{
A.S. Chernyatin, Yu.G. Matvienko, I.A. Razumovsky \\ Mechanical Engineering Research Institute of the Russian Academy of Sciences, Moscow, Russia
}

\begin{abstract}
Combining experimental and computational method for determination of the singular and the nonsingular stress terms along the front of the 3D surface crack is proposed. Evaluation of the terms is based on comprehensive comparison between deformation responses (for measurement points on the surface) obtained experimentally and from numerical solutions of the corresponding boundary problem of solid mechanics. The proposed approach allows carrying out an adequate and a comprehensive assessment of stress fields in the vicinity of the surface crack front.
\end{abstract}

KEYWORDS. 3D surface crack; Stress intensity factors; T-stresses; FEM; Minimization problem; Numerical experiment.

\section{INTRODUCTION}

$\mathrm{O}$ ne of the main trends in the development of methods for assessing of strength and lifetime of damaged structures, working under extreme loading conditions, is the application of fracture mechanics and, in particular, the methods for an analysis of the crack initiation process in the engineering structure. In this case, it is very important to estimate the singular and the non-singular stress terms in the Williams series solution along the crack front. It should be noted that these stress terms are dependent on crack and body configurations as well as loading conditions (e.g., $[1,2])$. At the same time, operational loading conditions of engineering components can strongly differ from design conditions due to crack- or notch-like defects, assembly and residual stress, etc.

To solve this problem, combining experimental and computational method can be employed for estimating operational loading conditions, the singular and the non-singular stress terms along the $3 \mathrm{D}$ surface crack front. The present paper deals with the approach which is based on comprehensive comparison between deformation responses (for measurement points on the surface of the engineering components) obtained experimentally and from numerical solutions of the corresponding boundary problem of solid mechanics. As a result, loading conditions, distribution of the singular $\left(K_{I}, K_{I I}\right)$ and the non-singular $\left(T_{x x}, T_{z}\right)$ terms along the surface crack front can be estimated.

\section{STATEMENT OF THE PROBLEM}

7 he object of research and numerical simulation (by means of FEM) is an elastic half-space with a semi-elliptical crack (length $2 a$, depth $b$ ) orthogonal to the surface. Homogeneous tensile stresses $\sigma_{x}, \sigma_{y}$ are applied in two orthogonal directions. Displacement fields on the surface for certain specimen configuration can be measured by electronic speckle interferometry (ESI) or computed by numerical experiment. It is necessary to estimate the stress intensity factor and the $T$-stress terms along the crack front by means of an analysis of these displacement fields. The problem can be solved by two steps. Firstly, the parameters $b, \sigma_{x}, \sigma_{y}$ should be estimated by the following procedure [3]:

$\checkmark$ generation of the experimental data $\left(e_{i}^{*}\right)$ for the displacement fields; 
creation so-called "response bank" which is mathematical analog of the FE model of the half-space with the surface crack. It allows calculating the values of the displacements in measurement points $\left(e_{i}\right)$ corresponding to $e_{i}^{*}$ in case arbitrary values of the searching parameters $\left(b, \sigma_{x}, \sigma_{y}\right)$;

$\checkmark$ solution of a minimization problem. Variation of the searching parameters is carrying out for achievement of the minimum of objective function which describes a discrepancy between $e_{i}^{*}$ and $e_{i}$;

$\checkmark$ assessment of $e_{i}^{*}$-scatter effect on determination accuracy of the searching parameters at different conditions of experimental measurements and minimization problem solution.

The second step is connected with calculation of stress fields in the vicinity of the crack front at the parameters $b, \sigma_{x}, \sigma_{y}$ which are defined by the first step. As results, distribution of the singular $\left(K_{I}, K_{I I}\right)$ and the non-singular $\left(T_{x x}, T_{p r}\right)$ terms along the crack front are calculated.

\section{NUMERICAL SIMULATION}

7 he proposed method is realized in the case of a numerical experiment. Generation of the original data for the displacement fields is performed by numerical simulation of the probe hole in the vicinity of the surface crack.

The finite element model of the elastic half-space under biaxial loading (Fig. 1a) is built in ANSYS software environment in the form of a prismatic body of finite dimensions, including the surface crack and volume of the probe hole (Fig. 1b). It should be noted that standard ANSYS procedure does not allow building a crack in this area, because its front is located over several volumes. Therefore, special multipurpose macros are created to build the planar crack with arbitrarily 3D-orientation and front geometry along which grid of singular elements is constructed in finite element model. Loading conditions are the following: $\sigma_{\mathrm{x}}=100 \mathrm{MPa}, \sigma_{\mathrm{y}}=200 \mathrm{MPa}$. The following sizes are also used in numerical simulation: the length of semi-elliptical crack $2 a=20 \mathrm{~mm}$ and the depth $b=(2 / 3) a=6.67 \mathrm{~mm}$, the diameter and depth of the probe hole $d=t=2 \mathrm{~mm}$.
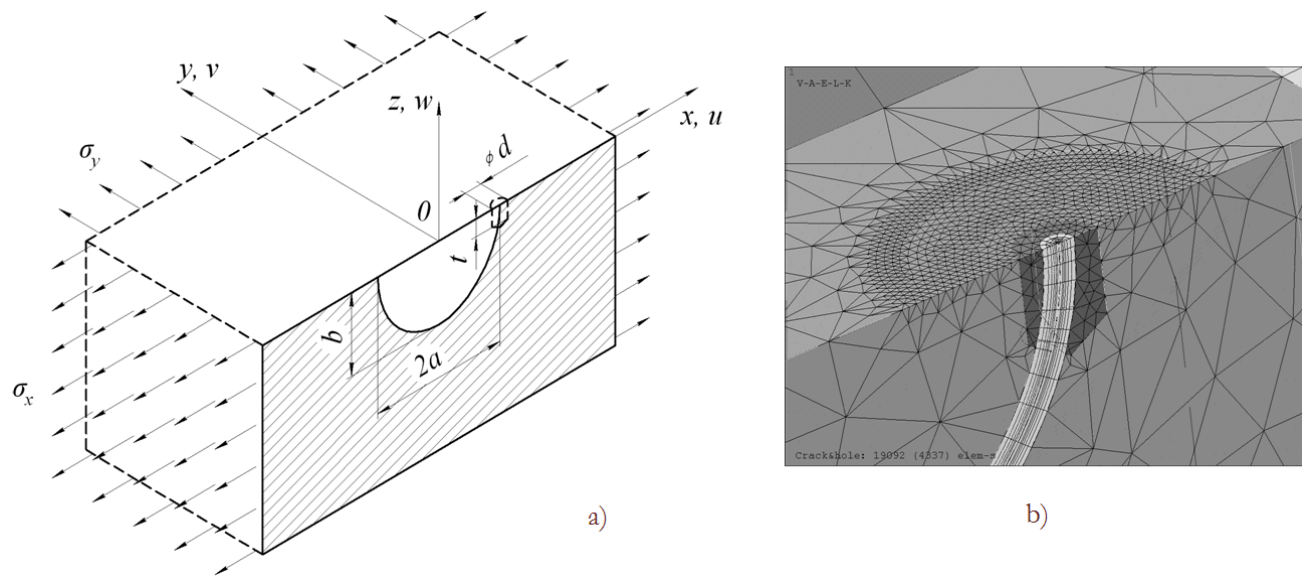

b)

Figure 1: The elastic half-space with a semi-elliptical crack and a probe hole (a) and finite element model of the output crack on the surface (b).

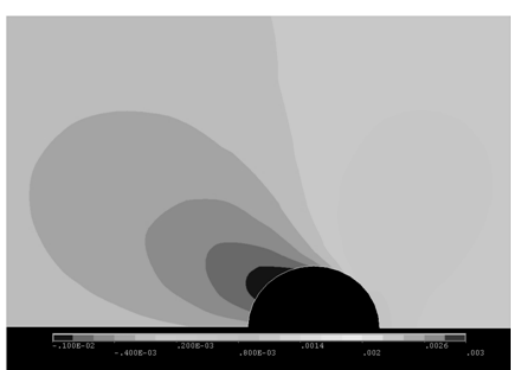

(a)

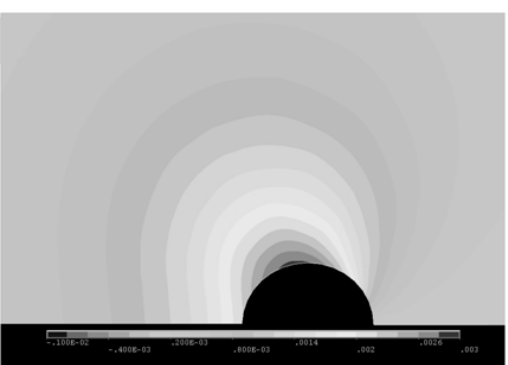

(b)

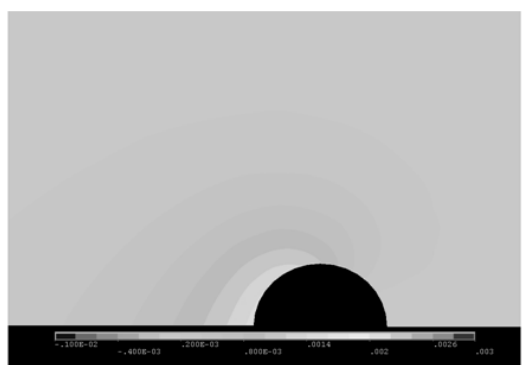

(c)

Figure 2: The displacement field u, v, w (a, b, c, respectively) due to the to the formation of the hole in the vicinity of the surface crack tip. 
The computed fields of tangential (u, v) and normal (w) displacement (Fig. 2), analogues of which in practice can be measured using the ESI, are obtained from the solution of the direct problem including calculation of stress and strain states due to the formation of the hole in the vicinity of the surface crack tip (Fig. 1a).

Further, the displacement fields $\mathrm{u}, \mathrm{v}, \mathrm{w}$ are used as the original experimental data to compute the strain response $e_{i}^{*}$ at corresponding points of the body under external load. The resulting array $e_{i}^{*}$ is employed for the calculation of parameters $P_{j}=\left\{b, \sigma_{x}, \sigma_{y}\right\}$ by minimization of the objective function $I\left(e_{i}^{*}, e_{i}\right)$ comparing experimental and numerical data. The details of the above-mentioned procedure are given in Ref. [3].

\section{ACCURACY OF THE RESULTS}

$\mathrm{T}$

he objective function $I\left(e_{i}^{*}, e_{i}\right)$ reached the minimum magnitude at the following condition $P_{j}=P_{j} *$. The objective function $I$ is taken as root-mean square norm $\left(\mathrm{I}_{\mathrm{RMS}}\right)$ or maximum norm $\left(\mathrm{I}_{\max }\right)$ reflecting the divergence between calculated $e_{i}$ and experimental $e_{i}^{*}$ data. The procedure of searching the minimum of the objective function is based on simplex method for function minimization [4] which is widely used in practice.

To analyze the stability of the solution and to assess the sensitivity of the procedure to the error of the experimental data, series of numerical experiments were carried out to determine the parameters $P_{j}$ under various conditions of experimental measurements and solution of the minimization problem. Variation of the experimental error $\left(\delta_{\mathrm{e}}\right)$, the number of measurement points $(N)$, and the area of their location (described by normalized radius vector $|\overline{\mathrm{r}}| / \varrho$, where $\rho$ is radius of the hole) is used to estimate the accuracy of the obtained results.

Some numerical results for the expectation and variation of the normalized parameters $\overline{\mathrm{b}}=\mathrm{b} / \mathrm{b}^{*}, \overline{\sigma_{\mathrm{x}}}=\sigma_{\mathrm{x}} / \sigma_{\mathrm{x}}^{*}, \overline{\sigma_{\mathrm{y}}}=\sigma_{\mathrm{y}} / \sigma_{\mathrm{y}}^{*}$ are summarized in Tab. 1. It can be seen that the proposed method gives very high accuracy.

\begin{tabular}{ccccccccccc}
\hline & \multicolumn{3}{c}{ Conditions } & \multicolumn{3}{c}{ Expectation } & \multicolumn{3}{c}{ Variation } \\
№ & $|\overline{\mathrm{r}}| / \varrho$ & $N$ & $\delta, \%$ & $I$ & $\overline{\mathrm{b}}$ & $\overline{\sigma_{x}}$ & $\overline{\sigma_{y}}$ & $\overline{\mathrm{b}}$ & $\overline{\sigma_{x}}$ & $\overline{\sigma_{y}}$ \\
1 & {$[2 ; 3]$} & 55 & 10 & $I_{\mathrm{RMS}}$ & 1.013 & 0.998 & 0.988 & 0.081 & 0.036 & 0.049 \\
2 & {$[2 ; 3]$} & 55 & 10 & $I_{\max }$ & 1.010 & 0.996 & 0.988 & 0.109 & 0.061 & 0.067 \\
3 & {$[1 ; 2]$} & 55 & 10 & $I_{\text {RMS }}$ & 1.049 & 0.994 & 0.982 & 0.216 & 0.091 & 0.116 \\
4 & {$[1 ; 2]$} & 55 & 10 & $I_{\max }$ & 1.075 & 0.989 & 0.975 & 0.262 & 0.093 & 0.128 \\
5 & {$[2 ; 3]$} & 110 & 10 & $I_{R M S}$ & 1.003 & 0.992 & 0.992 & 0.051 & 0.023 & 0.030 \\
6 & {$[2 ; 3]$} & 110 & 20 & $I_{\text {RMS }}$ & 1.030 & 0.970 & 0.959 & 0.124 & 0.055 & 0.071 \\
7 & {$[2 ; 3]$} & 110 & 20 & $I_{\max }$ & 1.054 & 0.093 & 0.932 & 0.097 & 0.040 & 0.055 \\
8 & {$[2 ; 3]$} & 60 & 20 & $I_{\text {RMS }}$ & 0.996 & 0.978 & 0.078 & 0.081 & 0.048 & 0.054 \\
\hline
\end{tabular}

Table 1: The influence of the experimental error on the values of the unknown parameters (numerical example).

\section{CALCULATION OF THE STRESS INTENSITY FACTORS AND THE T-STRESSES}

7 he final step of a comprehensive analysis is estimation of the distribution of the singular ( $\left.K_{I}, K_{I I}\right)$ and the nonsingular $\left(\mathrm{T}_{\mathrm{xx}}, \mathrm{T}_{\mathrm{zz}}\right)$ terms along the crack front on a basis of the calculated parameters $b, \sigma_{\mathrm{x}}, \sigma_{\mathrm{y}}$. The first and the second terms in a series expansion of the three-dimensional elastic stress components can be presented as follows

[5]:

$$
\begin{aligned}
& \sigma_{\mathrm{xx}}=\frac{1}{\sqrt{2 \pi \mathrm{r}}}\left[\mathrm{K}_{\mathrm{I}} \cos \frac{\theta}{2}\left(1-\sin \frac{\theta}{2} \sin \frac{3 \theta}{2}\right)-\mathrm{K}_{\mathrm{II}} \sin \frac{\theta}{2}\left(2+\cos \frac{\theta}{2} \cos \frac{3 \theta}{2}\right)\right]+\mathrm{T}_{\mathrm{xx}} \\
& \sigma_{\mathrm{yy}}=\frac{1}{\sqrt{2 \pi \mathrm{r}}}\left[\mathrm{K}_{\mathrm{I}} \cos \frac{\theta}{2}\left(1+\sin \frac{\theta}{2} \sin \frac{3 \theta}{2}\right)+\mathrm{K}_{\mathrm{II}} \sin \frac{\theta}{2}\left(\cos \frac{\theta}{2} \cos \frac{3 \theta}{2}\right)\right] \\
& \sigma_{\mathrm{xy}}=\frac{1}{\sqrt{2 \pi \mathrm{r}}}\left[\mathrm{K}_{\mathrm{I}} \sin \frac{\theta}{2}\left(\cos \frac{\theta}{2} \cos \frac{3 \theta}{2}\right)+\mathrm{K}_{\mathrm{II}} \cos \frac{\theta}{2}\left(1-\sin \frac{\theta}{2} \sin \frac{3 \theta}{2}\right)\right]
\end{aligned}
$$




$$
\begin{aligned}
\sigma_{\mathrm{zz}} & =\frac{2 v}{\sqrt{2 \pi \mathrm{r}}}\left[\mathrm{K}_{\mathrm{I}} \cos \frac{\theta}{2}-\mathrm{K}_{\mathrm{II}} \sin \frac{\theta}{2}\right]+\mathrm{T}_{\mathrm{zz}} \\
\mathrm{T}_{\mathrm{zz}} & =\mathrm{E} \varepsilon_{\mathrm{zz}}+v \mathrm{~T}_{\mathrm{xx}}
\end{aligned}
$$

Here, $K$ is the elastic stress intensity factor, $r$ and $\theta$ are the polar coordinates in plane x0y, $\varepsilon_{z z}$ is the strain in the $z$ direction, $E$ and $v$ are the Young modulus and Poisson's ratio, respectively. The terms $\mathrm{T}_{\mathrm{xx}}$ and $\mathrm{T}_{\mathrm{zz}}$ are the amplitudes of the second order terms in the three-dimensional series expansion of the crack-front stress field in the $\mathrm{x}$ - and $\mathrm{z}$-directions, respectively.

The stress intensity factor is calculated in a number of points (at variable values of $\mathrm{r} \leq \mathrm{a} / 20$ ) using the following equation

$$
\begin{aligned}
& \mathrm{K}_{\mathrm{I}}=\sqrt{\frac{\pi \mathrm{r}}{2}}\left(\left.2 \sigma_{\mathrm{xx}}\right|_{\theta=0}-\left.\sigma_{\mathrm{xx}}\right|_{\theta=-\pi}-\left.\sigma_{\mathrm{xx}}\right|_{\theta=+\pi}\right) \\
& \mathrm{K}_{\mathrm{II}}=\sqrt{\frac{\pi \mathrm{r}}{8}}\left(\left.\sigma_{\mathrm{xx}}\right|_{\theta=-\pi}-\left.\sigma_{\mathrm{xx}}\right|_{\theta=+\pi}\right)
\end{aligned}
$$

The obtained values of the stress intensity factor are extrapolated to the point $r=0$. To evaluate the distribution of the stress intensity factors along the crack front, this procedure is used for a number of orthogonal to the front of the crack plane $(\mathrm{x} 0 \mathrm{y})$. Their location is characterized by local coordinate salong the front which starts from the center of the crack front and finishes at the point located on the body surface.

The magnitude of the $T$-stress terms is defined through stress components for the points on the crack surface as follows

$$
\begin{aligned}
& \mathrm{T}_{\mathrm{xx}}=\frac{1}{2}\left[\left.\sigma_{\mathrm{xx}}\right|_{\theta=-\pi}+\left.\sigma_{\mathrm{xx}}\right|_{\theta=+\pi}\right] \\
& \mathrm{T}_{\mathrm{zz}}=\frac{1}{2}\left[\left.\sigma_{\mathrm{zz}}\right|_{\theta=-\pi}+\left.\sigma_{\mathrm{zz}}\right|_{\theta=+\pi}\right]
\end{aligned}
$$

The determination of the non-singular $\mathrm{T}_{\mathrm{xx}}$ - and $\mathrm{T}_{\mathrm{zz}}$-stress is similar to the calculation procedure for the stress intensity factor including extrapolation to the point $r=0$.

The distribution of the singular $\left(\mathrm{K}_{\mathrm{I}}, \mathrm{K}_{\mathrm{II}}\right)$ and the non-singular $\left(\mathrm{T}_{\mathrm{xx}}, \mathrm{T}_{\mathrm{zz}}\right)$ terms along the crack front (in the absence of the probe hole) is shown in Fig. 3. The stress intensity factor $K_{I I}$ is negligible as it is expected for the surface crack and loading conditions under consideration. It can be also seen that the non-singular terms are significantly changed along the surface crack front. In contrast to the $T_{\mathrm{xx}}$-stress, the $\mathrm{T}_{\mathrm{zz}}$-stress approaches the maximum value at the center of the crack front.

\section{ConClusions}

I $\mathrm{t}$ was shown that combining experimental and computational method can be employed for estimating operational loading conditions, the singular and the non-singular stress terms in a series expansion of the three-dimensional elastic stress components along the surface crack front. The proposed method is based on comprehensive comparison between deformation responses (for measurement points on the surface of the engineering components) obtained experimentally and from numerical solution of the corresponding boundary problem of solid mechanics. The distribution of the singular $\left(K_{I}, K_{I I}\right)$ and the non-singular $\left(T_{x x}, T_{z:}\right)$ terms along the surface crack front is computed.

\section{ACKNOWLEDGEMENT}

he authors acknowledge the support of the Russian Foundation of Basic Research (Grant N 12-08-91158NSFC_a). 

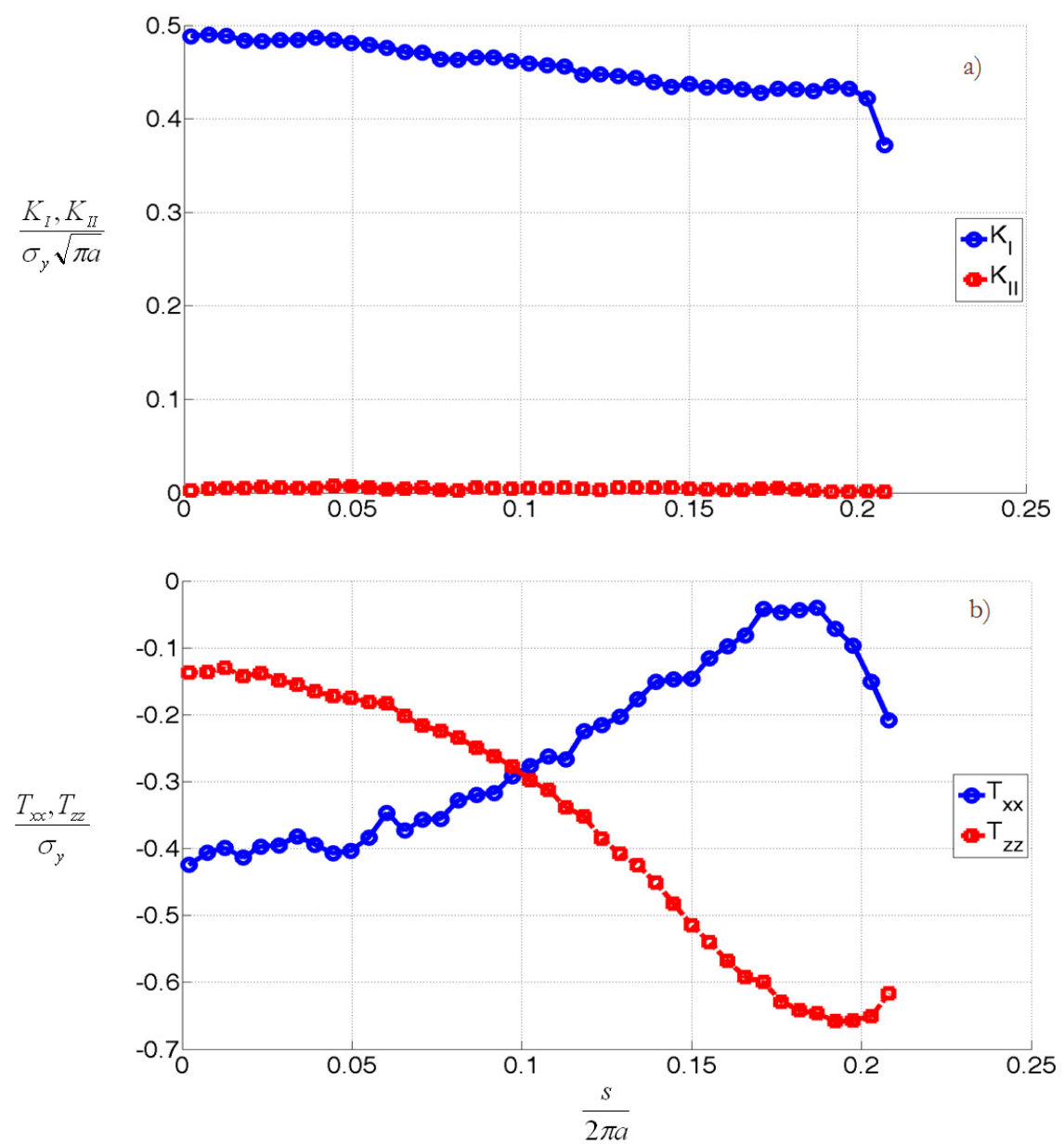

Figure 3: The distribution of the stress intensity factors (a) and the non-singular stresses (b) along the crack front.

\section{REFERENCES}

[1] Gonzalez-Albuixech, V.F., Giner, E., Fernandez-Saez, J., Fernandez-Canteli, A. Influence of the $t_{33}$-stress on the 3-D stress state around corner cracks in an elastic plate, Engng. Fract. Mech., 78 (2011) 412-427.

[2] Matvienko, Yu.G., Shlyannikov, V.N., Boychenko, N.V. In-plane and Out-of-plane Constraint Parameters along a Three-dimensional Crack-Front Stress Field under Creep Loading, Fatigue Fract. Engng. Mater. Struct., 36 (2013) 1424.

[3] Razumovsky, I.A., Chernyatin, A.S. Experimental numerical method to estimate loading conditions for structures with surface cracks, Journal of Machinery and Reliability, 3 (2009) 247-254.

[4] Nelder, J.A., Mead, R. A Simplex Method for Function Minimization. Computer Journal, 7 (1965) 308-313.

[5] Nakamura, T., Parks, D. M. Determination of elastic T-stress along three-dimensional crack fronts using an interaction integral, Int. J. Solids Struct., 29 (1992) 1597-1611. 